Revista Energia na Agricultura

ISSN 1808-8759

\title{
APLICAÇÃO DA TEORIA FUZZY NO CONTROLE DE SISTEMAS DE GERAÇÃO DE ENER- GIAS ALTERNATIVAS ${ }^{1}$ \\ FERNANDO DE LIMA CANEPPELE ${ }^{2} \&$ ODIVALDO JOSÉ SERAPHIM ${ }^{3}$
}

RESUMO: Este trabalho apresentou a aplicação e uso de uma metodologia fuzzy e simulou seu uso no controle inteligente de um sistema híbrido de geração de energia elétrica, utilizando as energias solarfotovoltaica e eólica. Quando se utiliza um sistema de controle fuzzy, é atingido o ponto de máxima geração de energia, transferindo desta forma toda a energia gerada a partir das fontes alternativas, solarfotovoltaica e eólica, à carga e/ou as baterias quando do seu uso não imediato. O modelo utilizado adotou três variáveis de entrada, que foram: velocidade do vento, radiação solar e carga do banco de baterias. Para variável de saída têm-se a escolha de qual das baterias do banco de baterias será carregada. Para as simulações deste trabalho foi utilizado o software MATLAB. Neste ambiente matemático computacional foram analisadas e simuladas todas as modelagens matemáticas, regras e demais variáveis descritas no sistema fuzzy. Este modelo pode ser usado em um sistema de controle de sistemas híbridos de geração de energia, proporcionando o melhor aproveitamento das fontes de energia, sol e vento, de modo que se possa extrair o máximo de energia possível dessas fontes alternativas sem nenhum prejuízo ao meio ambiente.

Palavras-chave: Teoria fuzzy, energia solar fotovoltaica, energia eólica.

\footnotetext{
${ }^{1}$ Parte da dissertação de mestrado do primeiro autor intitulada: DESENVOLVIMENTO DE UM MODELO FUZZY PARA OTIMIZAÇÃO DA ENERGIA GERADA POR UM SISTEMA HÍBRIDO (SOLAR-FOTOVOLTAICO E EÓLICO).

${ }^{2}$ Aluno do curso de PG Energia na Agricultura - FCA/UNESP - Botucatu/SP - Brasil. Docente no CEETEPS e na UNESP/Itapeva. fernando@itapeva.unesp.br

${ }^{3}$ Orientador e docente do Departamento de Engenharia Rural - FCA/UNESP - Botucatu/SP. seraphim@ fca.unesp.br
} 


\section{IMPLEMENTATION OF THE FUZZY THEORY IN CONTROL OF ALTERNATIVE ENERGY GENERATION SYSTEMS}

SUMMARY: This paper presents the application and use of a methodology based on fuzzy theory and simulates its use in intelligent control of a hybrid system for generating electricity, using solar energy, photovoltaic and wind. When using a fuzzy control system, it reached the point of maximum generation of energy, thus shifting all energy generated from the alternative sources-solar photovoltaic and wind, cargo and / or batteries when its use not immediately. The model uses three variables used for entry, which are: wind speed, solar radiation and loading the bank of batteries. For output variable has to choose which of the batteries of the battery bank is charged. For the simulations of this work is used MATLAB software. In this environment mathematical computational are analyzed and simulated all mathematical modeling, rules and other variables in the system described fuzzy. This model can be used in a system of control of hybrid systems for generating energy, providing the best use of energy sources, sun and wind, so we can extract the maximum energy possible these alternative sources without any prejudice to the environment .

Keywords: Fuzzy theory, solar photovoltaics, wind power.

\section{INTRODUÇÃO}

As energias solar e eólica são fontes renováveis e não poluentes, constituindo recursos alternativos opcionais para geração de energia elétrica. Muitos países, com velocidades médias de vento na faixa de 5 a $10 \mathrm{~ms}^{-1}$, estão utilizando sistemas de conversão da energia eólica em energia elétrica (geradores eólicos), em um esforço para minimizar a dependência de combustíveis fósseis que não são renováveis (BELLARMINE, 1996). Atualmente milhares de sistemas fotovoltaicos, em regiões com radiação solar média diária na faixa de 3 a $6 \mathrm{kWhm}^{-2}$, estão instalados ao redor do mundo, provendo pequenas potências, aplicações em redes independentes ou em sistemas individuais em regiões isoladas (MAHMOUD, 1990).

Por outro lado, uma desvantagem comum para as energias eólica e solar reside na dependência das variações climáticas. Ambas as formas de energia, se usadas independentemente, teriam que ser superdimensionadas, para tornarem-se confiáveis, resultando em um custo total muito mais elevado. Porém, uma combinação da energia solar e eólica em um sistema de geração híbrido pode atenuar as flutuações individuais destas formas de energia, aumentando a produção de energia global e reduzindo significativamente a 
necessidade de armazenamento de energia. Devido a esta combinação, a despesa global para sistemas autônomos pode ser reduzida drasticamente para um grande número de casos (BAGUL, 1996).

Modelos matemáticos implantados em programas computacionais têm apresentado significativos resultados em sistemas não lineares e complexos nas mais diversas áreas, entre as quais, a teoria de controle, modelo de reconhecimento e análise de decisão. Esses programas são capazes de resolver questões que os modelos clássicos, via de regra, não são capazes de fazê-lo. São os chamados de sistemas inteligentes, dentre os quais se destacam as Redes Neurais e a Lógica Fuzzy (SOUZA, 2004).

Informações vagas, incertas, qualitativas, comunicações verbais, capacidade de aprendizado e de formulação de estratégias de tomadas de decisão são características humanas. Portanto, a teoria fuzzy é freqüentemente referida como "inteligente", devido ao fato de simular a inteligência humana (MCNEILL e FREIBERGER, 1994).

Neste contexto, este trabalho teve o objetivo específico de descrever o uso da teoria fuzzy para o controle de um sistema híbrido de geração de energia eólico-fotovoltaico de pequeno porte no suprimento de energia requerida por uma residência de uma propriedade rural, tirando o máximo de energia das variáveis meteorológicas sobre a produção de energia elétrica pelo sistema.

\section{MATERIAL E MÉTODOS}

O trabalho foi desenvolvido no Núcleo de Energias Alternativas e Renováveis - NEAR do Laboratório de Energização Rural do Departamento de Engenharia Rural da UNESP, Faculdade Ciências Agronômicas, Fazenda Experimental Lageado, localizada no município de Botucatu, São Paulo, com localização geográfica definida pelas coordenadas $22^{\circ} 51^{\prime}$ Latitude Sul (S) e $48^{\circ} 26^{\prime}$ Longitude Oeste (W) e altitude média de 786 metros acima do nível do mar. A velocidade média mensal do vento a $10 \mathrm{~m}$ de altura é 3,1 ms-1 e a energia solar global média mensal diária é de 4772,13 Whm-2 (SILVA, 2000).

\subsection{Dados meteorológicos e elétricos}

Neste trabalho foram utilizados dados obtidos pela estação acima mencionada. Seguem abaixo nas Tabelas 1 e 2, exemplos de dados meteorológicos coletados e já integralizados de acordo com Siquei$\operatorname{ra}(2005)$. 
Tabela 1 - Características da energia eólica, velocidade do vento no período de 01/07 a 31/07 de 2005.

\begin{tabular}{lll}
\hline Dia/Mês & Velocidade do Vento Média Diária $\left(\mathrm{ms}^{-1}\right)$ & Energia Disponível no Vento Total Diária (Wh) \\
\hline $1 / 7$ & 3,5 & 2,2 \\
\hline $2 / 7$ & 2,6 & 19,5 \\
\hline $3 / 7$ & 4,5 & 420,5 \\
\hline $4 / 7$ & 2,8 & 4,7 \\
\hline$\ldots$ & $\ldots$ & $\ldots$ \\
\hline $28 / 7$ & 0,0 & 0,0 \\
\hline $29 / 7$ & 3,0 & 29,7 \\
\hline $30 / 7$ & 2,6 & 111,2 \\
\hline $31 / 7$ & 2,1 & 22,5 \\
\hline
\end{tabular}

Observa-se que não há uma relação direta e proporcional da velocidade do vento com a energia disponível no vento. Esta se deve à característica intermitente do vento, rajadas e outros fatores que fazem com que nem todo vento venha a gerar energia. Outra condição importante e que pode limitar a geração eólica é o fato de a bateria estar carregada. Se estiver, então o aerogerador automaticamente para de funcionar. Este fato é uma das justificativas para o uso de mais baterias e o gerenciamento através de um controlador fuzzy.

Tabela 2 - Características da energia solar, irradiância no período de 01/07 a 31/07 de 2005.

Dia/Mês Irradiância Solar Média Diária $\left(W \cdot m^{-2}\right)$ Energia Solar Global Total Diária (Wh)

\begin{tabular}{lll}
\hline $1 / 7$ & 610,3 & $13.293,2$ \\
\hline $2 / 7$ & 582,8 & $12.693,1$ \\
\hline $3 / 7$ & 275,3 & $5.698,0$ \\
\hline $4 / 7$ & 164,0 & $3.600,8$ \\
\hline$\cdots$ & $\cdots$ & $\cdots$ \\
\hline $28 / 7$ & 463,8 & $10.770,5$ \\
\hline $29 / 7$ & 626,1 & $14.426,3$ \\
\hline $30 / 7$ & 626,2 & $14.427,4$ \\
\hline $31 / 7$ & 617,2 & $13.886,4$ \\
\hline
\end{tabular}


Para o projeto do controlador fuzzy, deve-se utilizar uma faixa de valores ou limites préestabelecidos. Da análise das tabelas 1 e 2, pode-se definir estes valores em relação aos dados ambientais que foram considerados (radiação solar e velocidade do vento). Os limites superiores e inferiores de acordo com dados obtidos da estação meteorológica do NEAR e que tem potencial para geração de energia elétrica conforme Siqueira (2005), estão descritos na Tabela 3:

Tabela 3 - Limites superiores e inferiores para Radiação Solar Global e Velocidade do Vento

\begin{tabular}{lll}
\hline & Limite Inferior & Limite Superior \\
\hline Radiação Solar Global & $300 \mathrm{~W} / \mathrm{m} 2$ & $1200 \mathrm{~W} / \mathrm{m} 2$ \\
\hline Velocidade do Vento & $3 \mathrm{~m} / \mathrm{s}$ & $12 \mathrm{~m} / \mathrm{s}$ \\
\hline
\end{tabular}

\subsection{Simulações através de programas matemáticos}

Para as simulações referentes ao uso da teoria fuzzy para controle, utilizou-se o ambiente de computação científica MATLAB e estas simulações foram realizadas junto ao Departamento de Engenharia Elétrica da FEB-UNESP e junto ao Departamento de Matemática da FC-UNESP, ambos localizados no campus da UNESP em Bauru-SP.

Diversos usos distintos podem ser feitos por meio do MATLAB, dentre eles o uso de várias caixas de ferramentas, cada qual destinada a tratar diferentes classes de problemas científicos. Dentre estas, foi utilizada neste trabalho o Fuzzy Logical Toolbox.

\subsection{Modelagem do controlador fuzzy para o sistema híbrido}

Seguindo a sistemática proposta para o desenvolvimento de um controlador fuzzy, a primeira etapa a ser realizada é a conversão das variáveis de entrada e saída em uma representação conhecida como conjuntos fuzzy e denomina-se fuzzyficação.

Inúmeros são os tipos de funções de pertinência que podem ser utilizadas, mas neste trabalho optou-se por uma combinação das funções triangular e trapezoidal devido a sua facilidade de geração e excelente precisão nos resultados, além de estarem disponíveis para pronta utilização na maioria das ferramentas matemáticas computacionais. Também será ressaltado que a utilização de outra função de pertinência não resultará em grandes vantagens. 
A segunda etapa estabelece as regras que relacionarão as variáveis de entrada e saída. Essas regras são obtidas do conhecimento e da experiência humana e denomina-se base de regras.

A terceira etapa é denominada de fuzzyficação e efetua a conversão dos conjuntos fuzzy em um número que representa o estado da saída do sistema para uma determinada condição.

\subsubsection{Fuzzyficação e grau de pertinência}

Utilizando o MATLAB, definiu-se as funções de pertinência associadas às variáveis de entrada (Velocidade do Vento e Radiação Solar) do controlador proposto, conforme as Figuras 1 e 2:

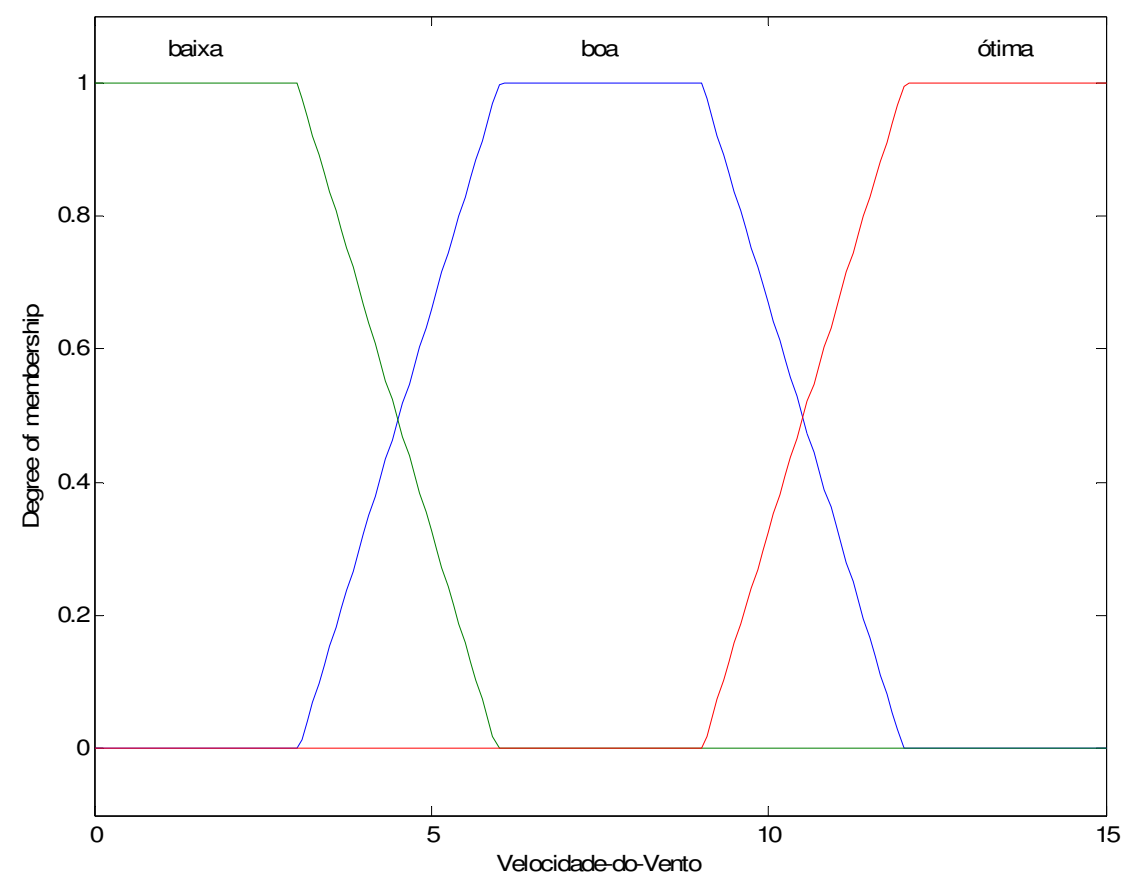

Figura 1 - Função de pertinências associada a variável de entrada "Velocidade do Vento". 


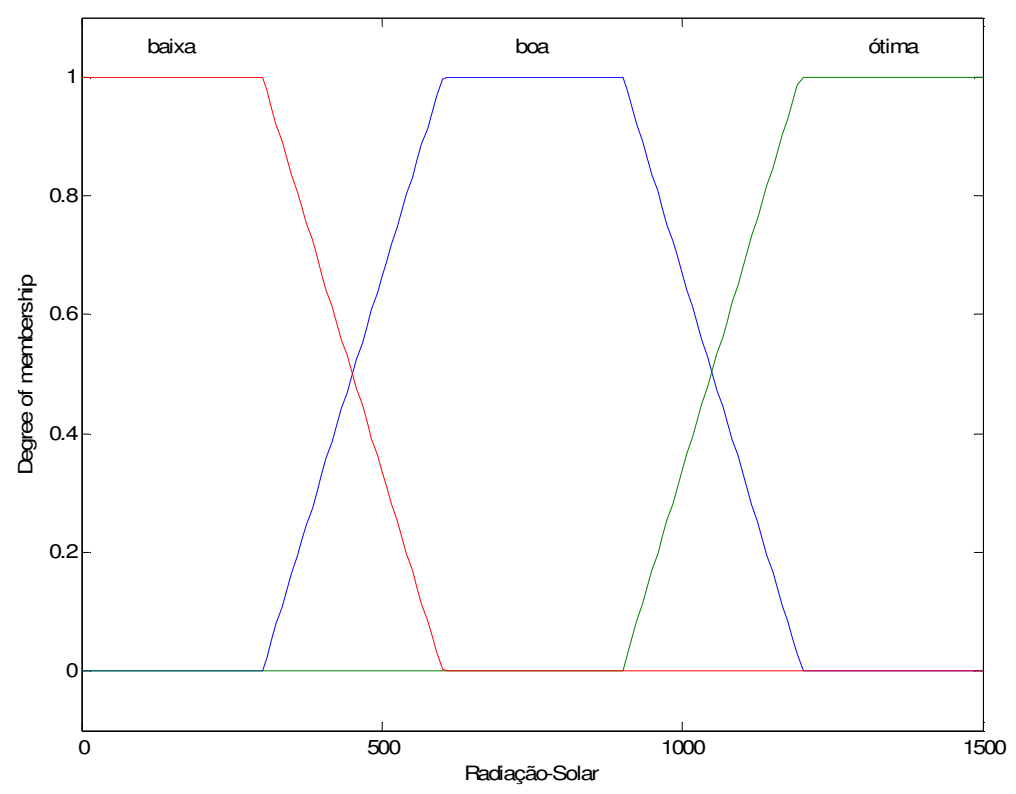

Figura 2 - Função de pertinências associada a variável de entrada "Radiação Solar".

As funções de pertinência associadas às variáveis de entrada (Carga das Baterias - A, B e C) do controlador proposto, podem ser observadas conforme mostra a Figura 3:

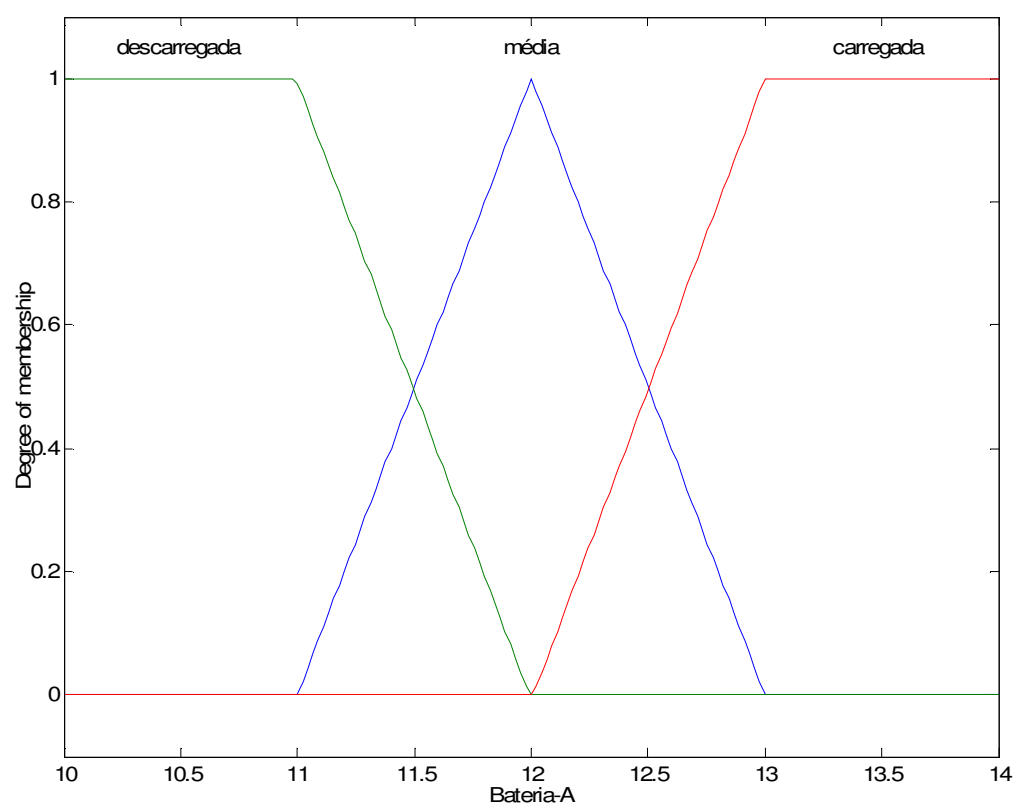

Figura 3 - Função de pertinências associada a variável de entrada "Carga da Bateria - A" 
Para as outras variáveis de entrada, "Cargas da Bateria - B e Carga da Bateria - C", tem-se as mesmas funções de pertinências mostradas na Figura 3.

$\mathrm{Na}$ Figura 4, tem-se as funções de pertinência relativas variável de saída (Carga do Banco de Baterias) do controlador fuzzy:

\subsubsection{Regras}

O mapa de regras é elaborado durante o projeto do sistema em si, identificando todas suas características e detalhes e, auxilia na identificação das decisões a serem tomadas durante a operação do processo. Ele é preenchido com o auxílio de um especialista, ou seja, um operador humano do sistema a ser controlado.

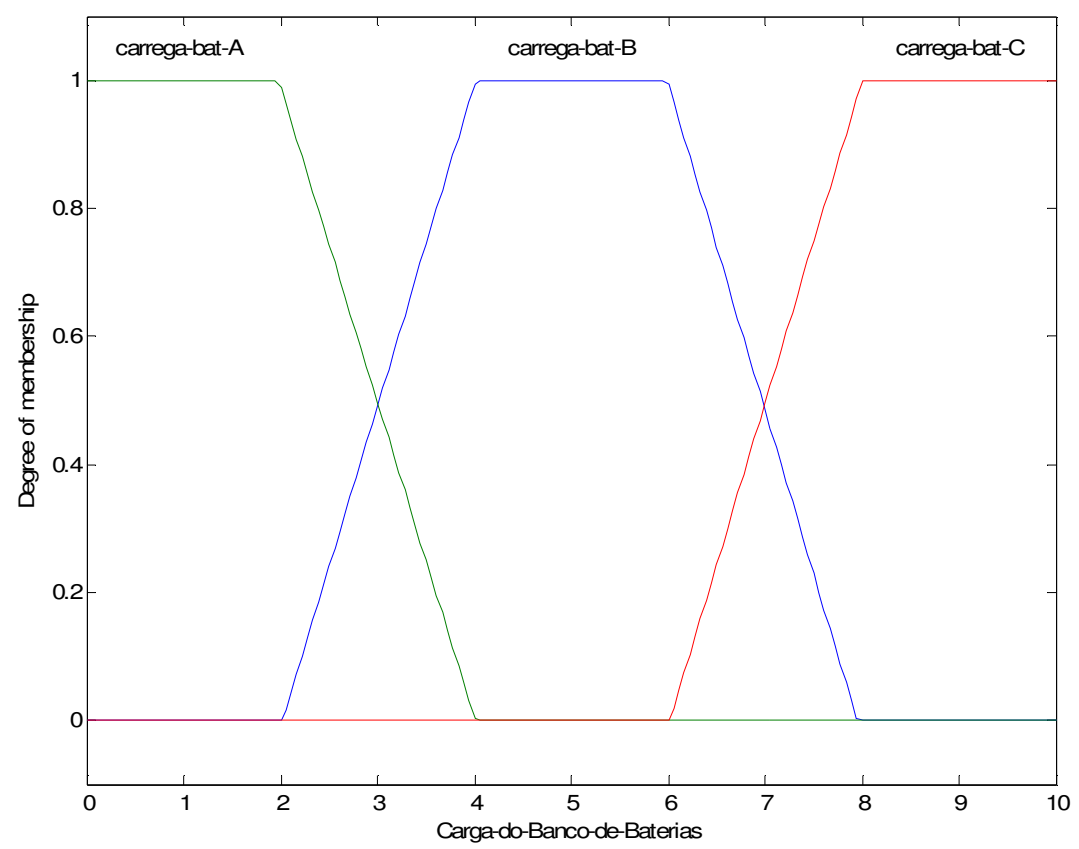

Figura 4 - Função de pertinências associada à variável de saída "Carga do Banco de Baterias".

Utilizando as teorias da lógica fuzzy, este mapa de regras pode ser gerado utilizando estrutura computacional do tipo: SE (Condição) ENTÃO (Conseqüência). 
As regras são escritas no editor de regras do Fuzzy Logical Toollbox do MATLAB que disponibiliza vários tipos de operações entre as funções de pertinência fuzzy e ainda permite que sejam criados novos tipos de funções de pertinência. Este procedimento é representado na Figura 5.

A partir de uma regra estabelecida, vão se repetindo os procedimentos de acordo com a entrevista ao especialista, colocando-se todas as situações que as variáveis podem apresentar e como o controlador dará a resposta de acordo com as entradas.

Uma vez determinadas e inseridas as regras, pode-se visualizá-las através do software citado para possíveis ajustes e correções, conforme ilustrado na Figura 6.

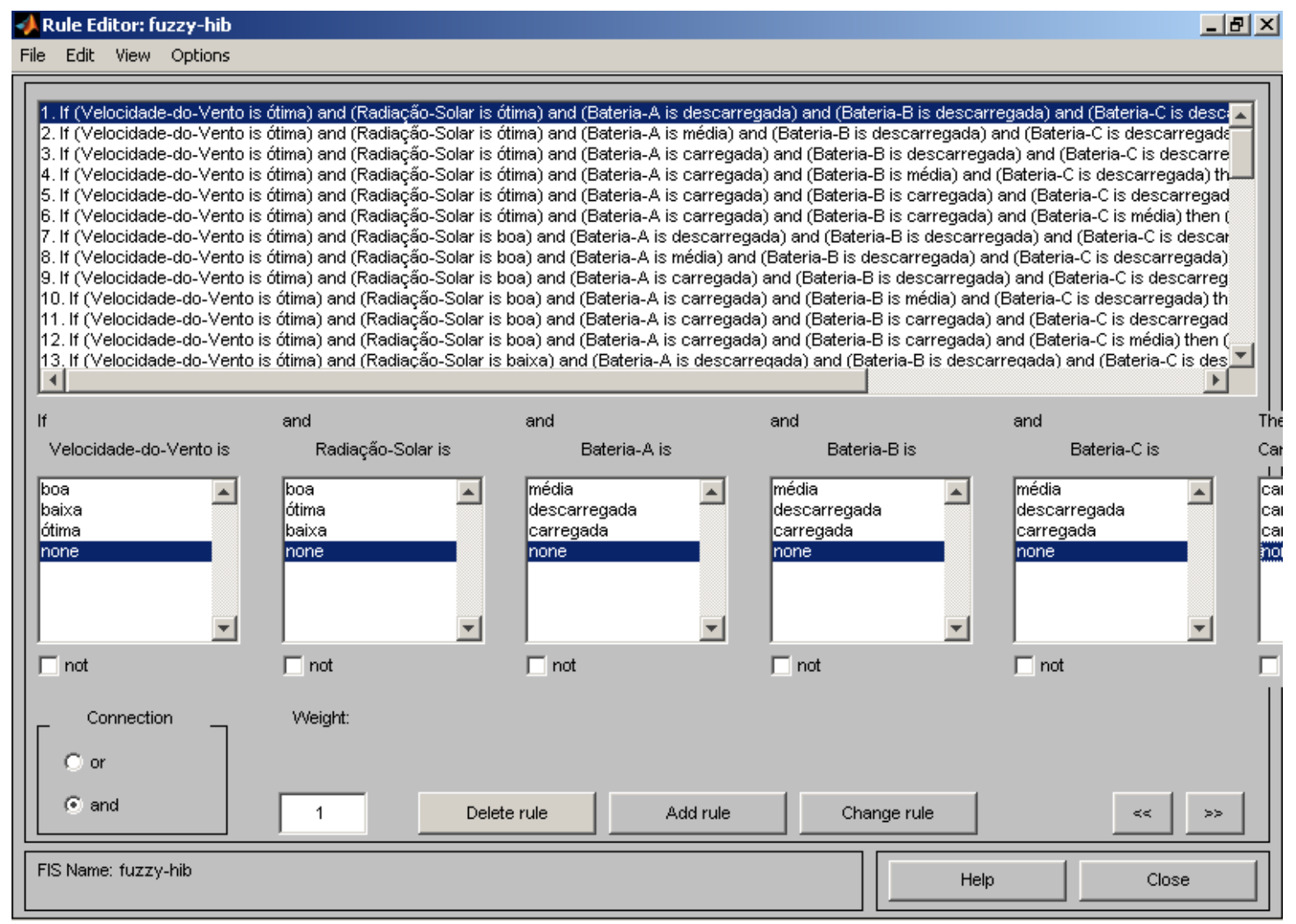

Figura 5 - Determinação e inserção das Regras Fuzzy para o sistema de controle.

Verifica-se que é através das regras que as funções de pertinência são ativadas, considerando-se seu grau em relação a cada conjunto fuzzy. Outra característica é que as regras fuzzy são agrupadas em blocos. Os chamados blocos de regras são parte fundamental da base de conhecimento dos controladores fuzzy. O controlador fuzzy recorre à verificação das regras para a tomada de decisão. 


\subsubsection{Defuzzyficação}

O passo final consiste em se efetuar a defuzzificação que será traduzir para um valor discreto o resultado da variável lingüística de saída do controlador que foi inferida pelas regras fuzzy. De uma forma genérica, este processo nada mais é que uma transformação inversa que traduz a saída do domínio fuzzy para o domínio discreto. Alguns métodos de defuzzificação são utilizados, entre eles citam-se: o centro da área, centro do máximo e a média do máximo. O processo mais utilizado é o primeiro, freqüentemente chamado de centro de gravidade, pois ele calcula o centróide da área que é composta pelo resultado das operações entre os conjuntos fuzzy.

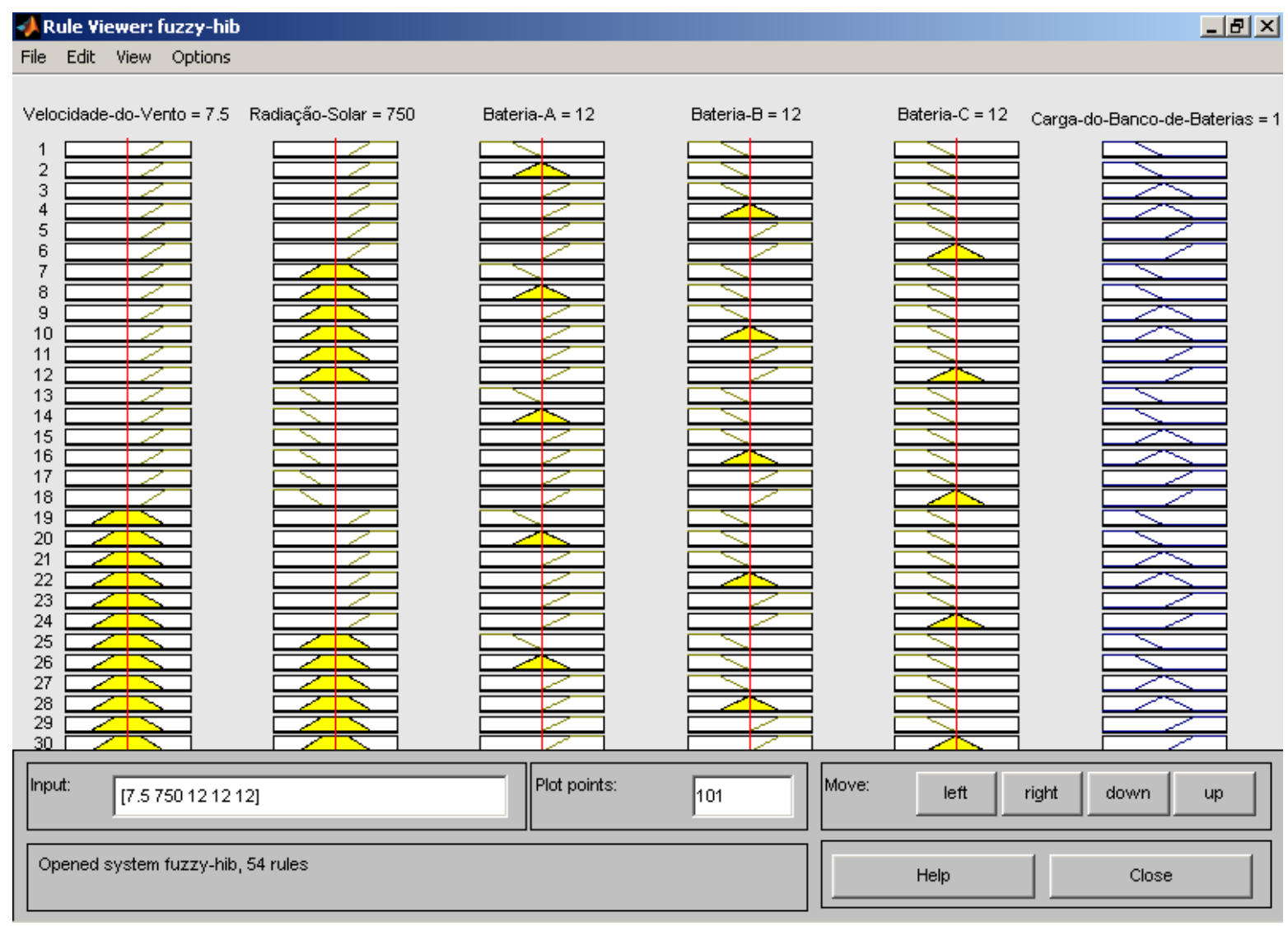

Figura 6 - Visualização gráfica das regras fuzzy. 


\section{RESULTADOS E DISCUSSÃO}

O controlador proposto deve possuir um comportamento o mais semelhante possível ao de um controlador humano. Dessa forma, a teoria fuzzy demonstrou ser útil na elaboração de um modelo de um sistema de controle para geração híbrida de energia.

Segundo Shaw e Simões (2001), o poder associado à utilização de teoria fuzzy é servir de ponte entre o conhecimento empírico e uma implementação computacional.

Desta forma, após as pesquisas, estudos e simulações, além de levar em consideração o estado da arte dos sistemas que utilizam teoria fuzzy para controle, chegou-se ao controlador como ilustrado da Figura 7. Esta figura é gerada automaticamente pelo MATLAB.

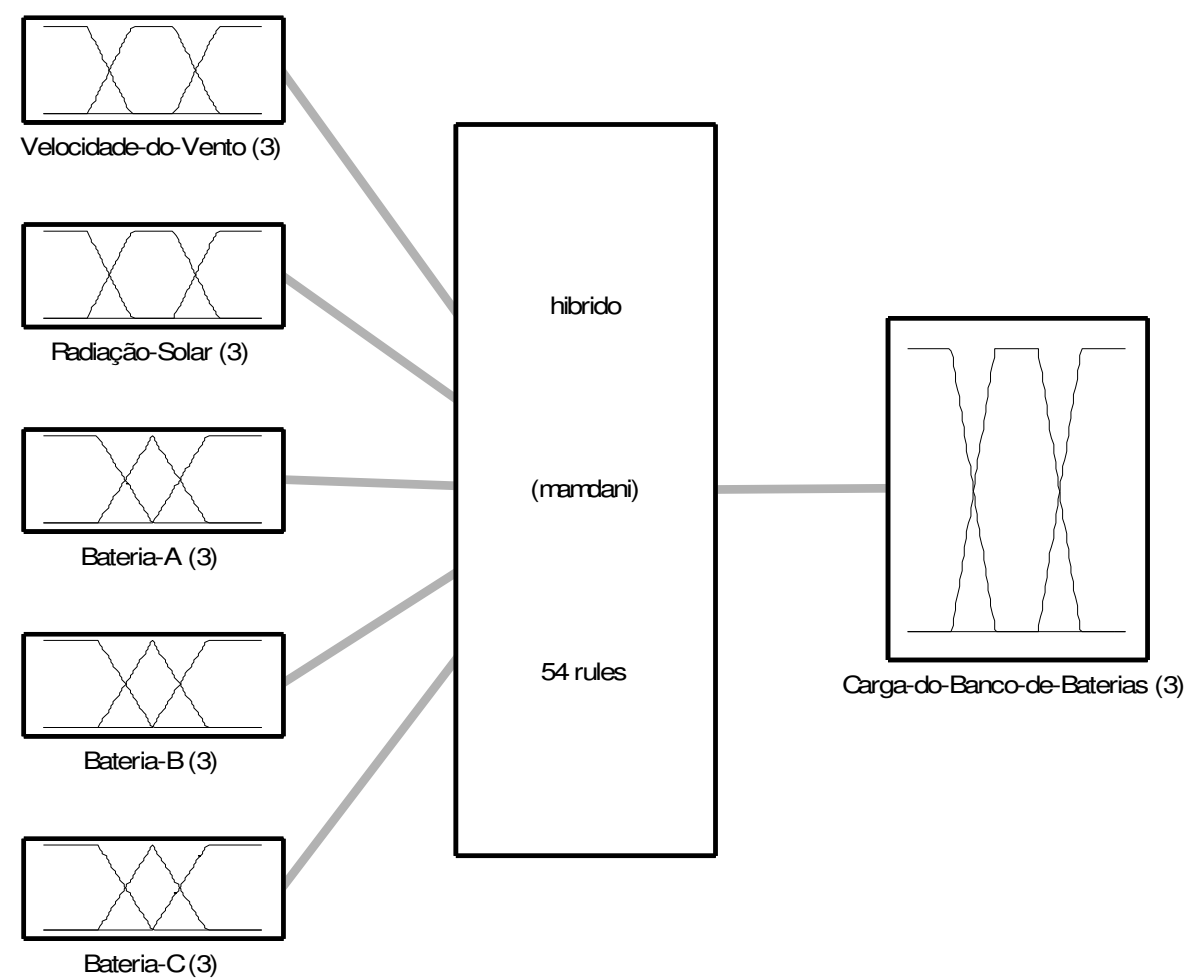

Systemhibrido: 5 inputs, 1 outputs, 54 rules

Figura 7 - Resumo do sistema de controle fuzzy para um sistema híbrido de geração de energia (solarfotovoltaica e eólica). 
Conforme descrito anteriormente, tem-se então o sistema de controle proposto já em funcionamento no MATLAB. De acordo com a Figura 7, o sistema tem 5 entradas, 1 saída e 54 regras que determinam seu comportamento.

A Figura 8 mostra a tela de configuração, edição e inserção de dados do Fuzzy Logical Toolbox do MATLAB para o sistema ilustrado na Figura 7.

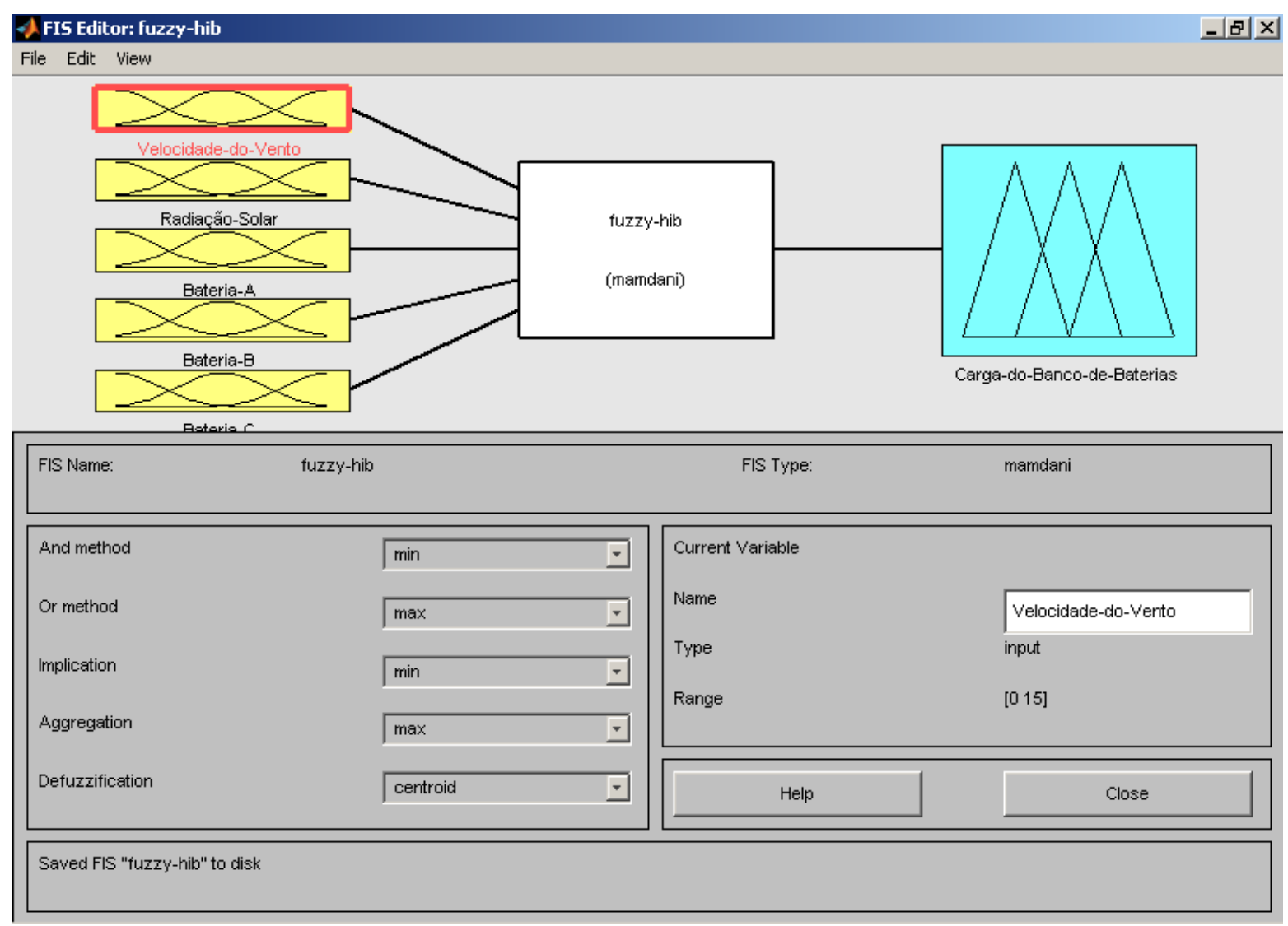

Figura 8 - Tela inicial e de configuração básica do Fuzzy Logical Toolbox do MATLAB.

Nas Figuras 9 e 10, tem-se o exemplo da tela onde se pode definir o domínio, a escolha do formato e número de funções de pertinência das variáveis de entrada e/ou saída.

A base de conhecimento do Controlador Fuzzy é composta por funções de pertinência, que compõem sua base dados, e por implicações verbais que compõem sua base de regras. 


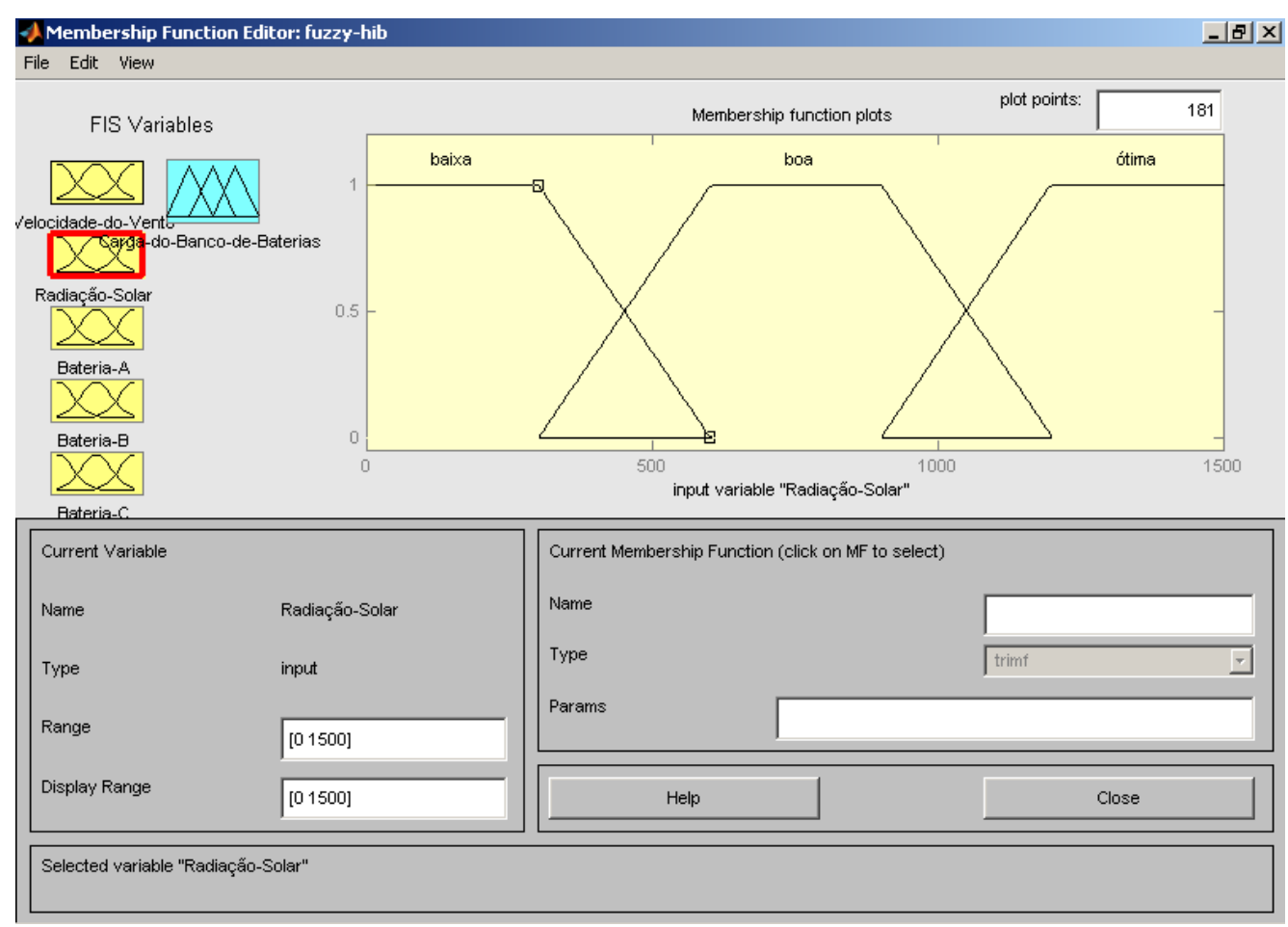

Figura 9 - Definição do domínio e escolha das funções de pertinência das variáveis de entrada.

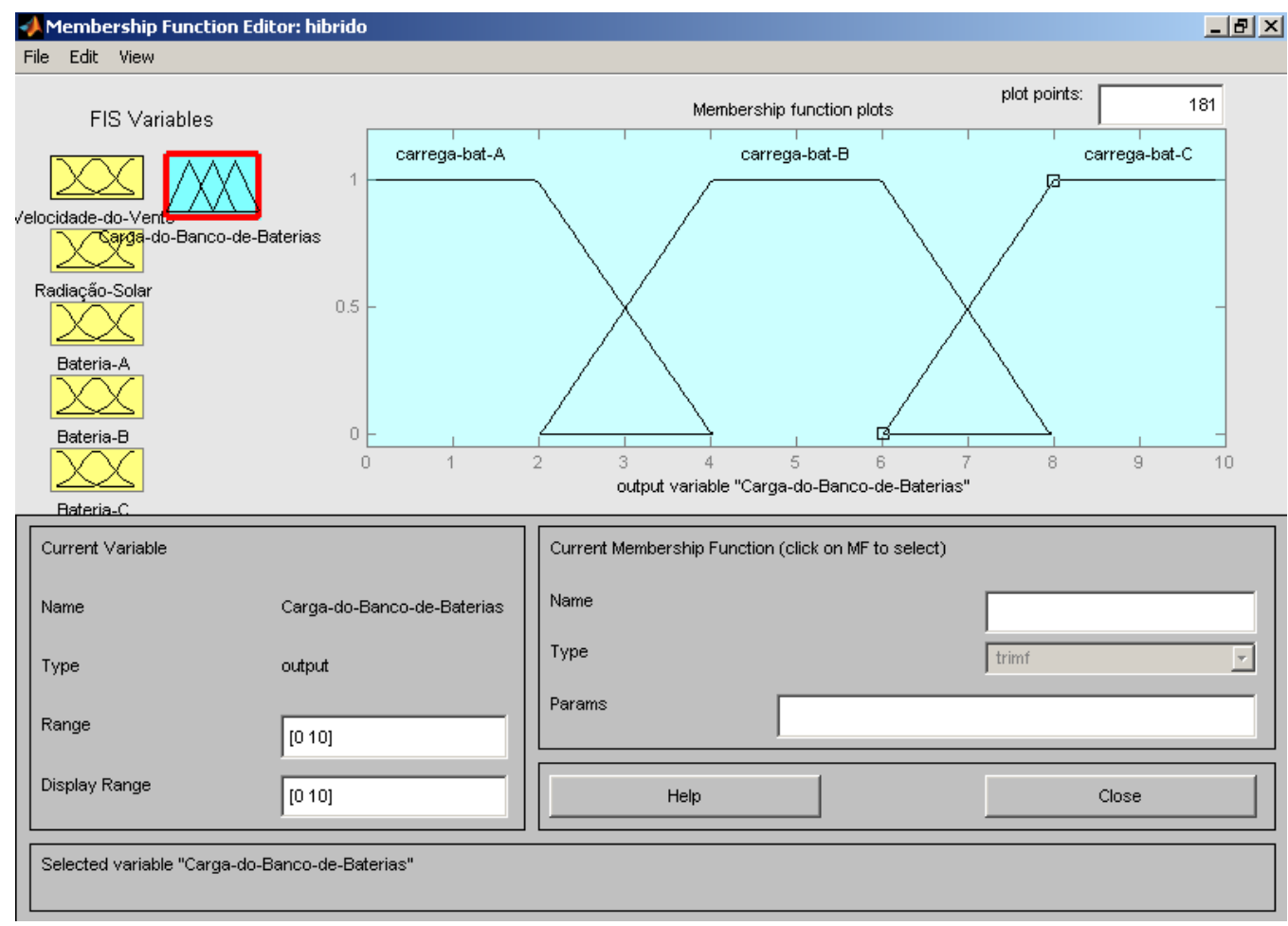

Figura 10 - Definição do domínio e escolha das funções de pertinência das variáveis de saída. 
Em um sistema de controle baseado na teoria fuzzy determinam-se as regras de acordo com o número de variáveis de entrada e de saída. Inúmeras regras podem ser estabelecidas para se controlar o sistema e sua inserção no sistema proposto está ilustrado na Figura 11.

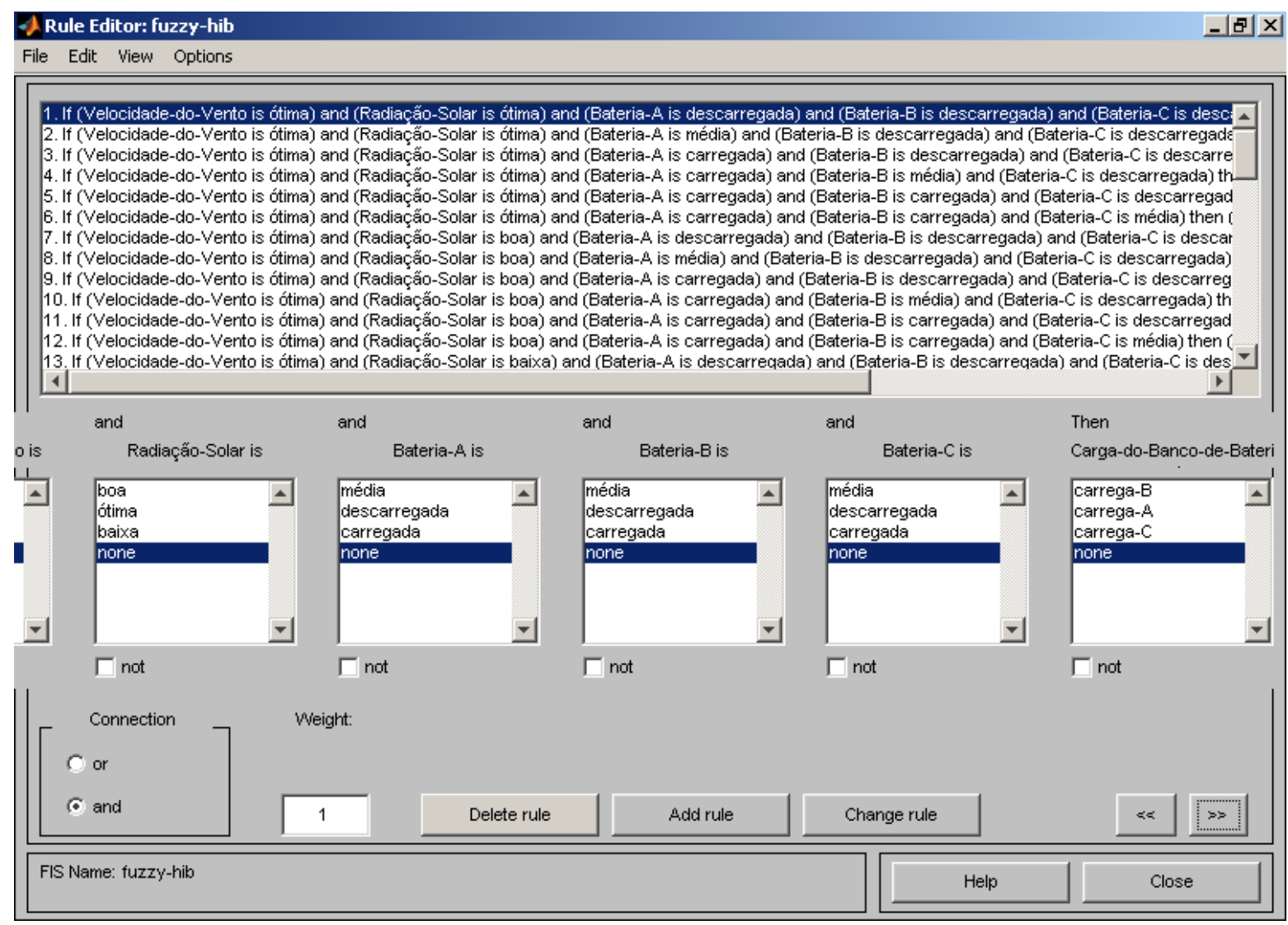

Figura 11 - Base de regras.

Na construção de cada regra deve-se definir a conexão entre as variáveis de entrada e as variáveis de saída, através dos operadores lógicos. Após a inserção de todas as regras tem-se a formação do sistema de controle fuzzy.

Como exemplo de funcionamento do controlador, pode-se atribuir para o sistema proposto, os seguintes valores as variáveis de entrada do sistema:

- Velocidade do vento: $10 \mathrm{~m} / \mathrm{s}$; Radiação solar: $1000 \mathrm{~W} / \mathrm{m} 2$;

- Carga da Bateria A: 13 V ; Carga da Bateria B: 13 V ; Carga da Bateria C: 10,5 V

Na Figura 12 tem-se os valores inseridos no controlador conforme o exemplo e a sua resposta através da variável de saída: 


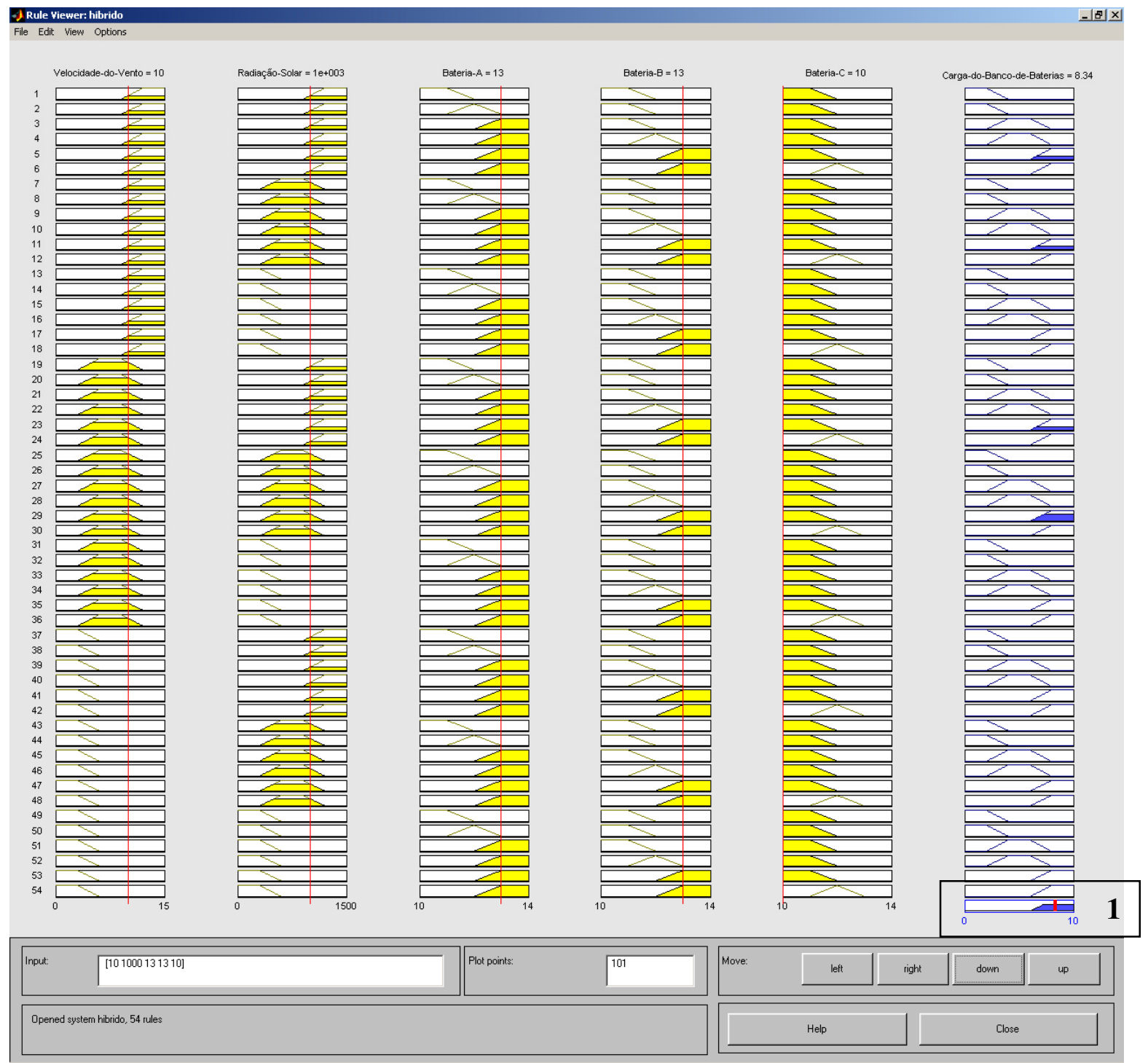

Figura 12 - Exemplo de simulação do controlador. Variáveis de entrada e de saída. 1 - Valor numérico de saída $(8,34)$.

A verdadeira saída defuzzyficada do controlador fuzzy pode ser considerada como passível de assumir somente dois valores: SIM ou NÃO, pois o sistema está sendo proposto para auxiliar em uma tomada de decisão.

Dessa maneira, de acordo com a Figura 12, aplicando-se o método do centro de gravidade, conforme anteriormente citado, tem-se um valor numérico de saída $(8,34)$, que nada mais é que a variável ainda no domínio Fuzzy. Observando novamente a Figura 4, pode-se fazer a "tradução" dessa variável de 
saída do domínio fuzzy para o domínio. O resultado será a ordem do controle para que se inicie a Carga da Bateria $\mathrm{C}$ e assim se completa a defuzzificação e conseqüente tomada de decisão.

Este valor fica mais evidente e claro quando se analisa a Figura 13, que trata da carga do banco de baterias.

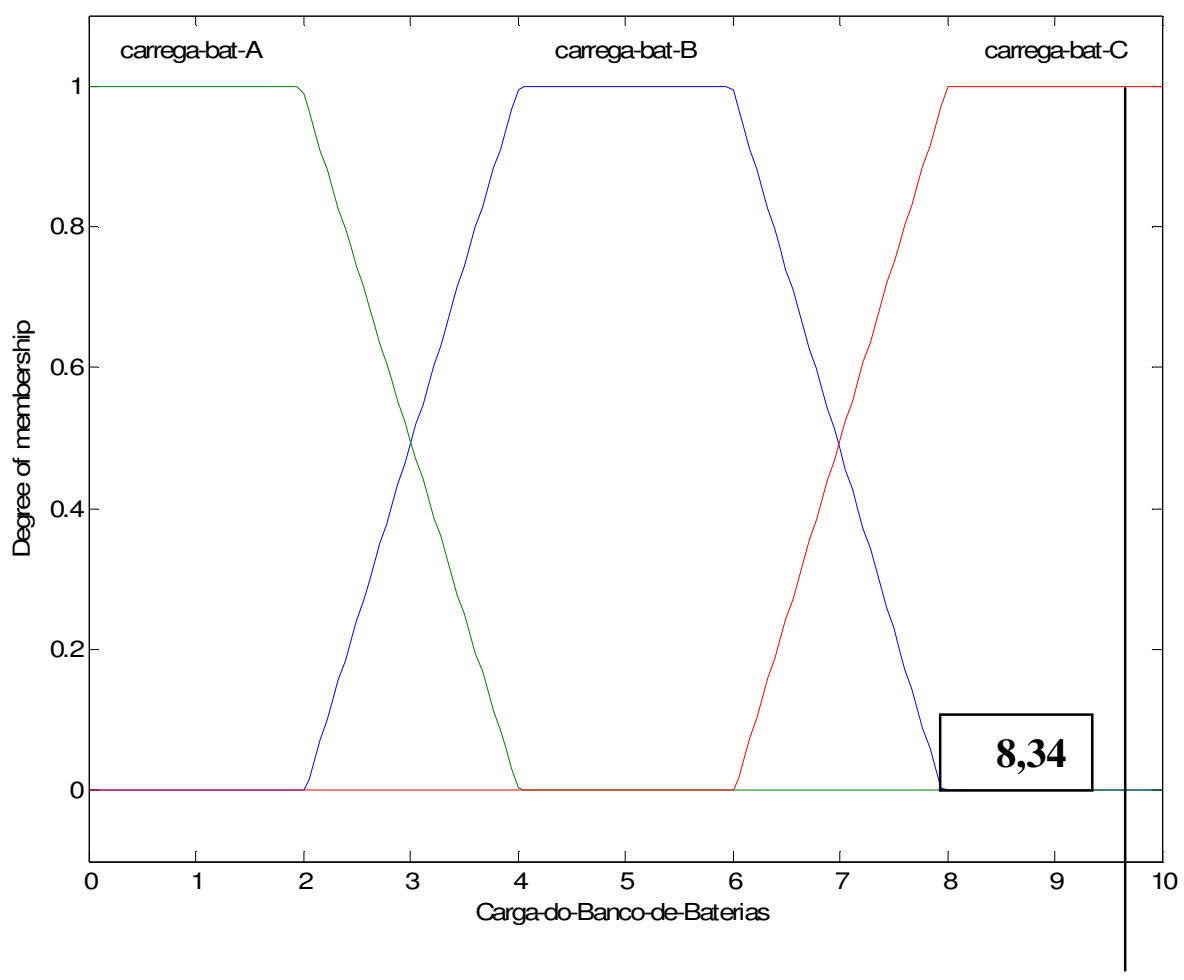

Figura 13 - Exemplo de simulação do controlador. Variável de saída e significado do valor numérico de saída $(8,34)$.

Este valor significa que o sistema irá direcionar a energia produzida para a carga da bateria C.

\section{CONCLUSÕES}

A utilização da teoria fuzzy permite identificar e implementar em um controlador a experiência do operador e sua forma de decidir sobre o problema em questão. Verifica-se a capacidade de operadores de processos de naturezas diversas realizarem com sucesso inferências sobre o mesmo de forma intuitiva, sem que seja necessário conhecer os valores numéricos das variáveis envolvidas. Um operador considera- 
do experiente acumula um maior número de observações anteriores que utiliza na formulação de previsões sobre o processo.

Desta forma, a grande vantagem da utilização da teoria fuzzy é a possibilidade de modelar e manipular matematicamente informações vagas, imprecisas e intermitentes, naturais da linguagem humana, e que são fornecidas pelos especialistas, não matemáticos, para caracterizar os processos estudados.

Esta manipulação pode ser facilmente feita a partir da junção das variáveis escolhidas para modelar matematicamente o sistema proposto, quando a implicação das variáveis independentes nas dependentes é estabelecida por um conjunto de regras lingüísticas baseadas no conhecimento de especialistas.

O que se pode constatar é que o controle fuzzy, que é uma das partes mais aprofundadas da teoria de conjuntos fuzzy, possibilita-nos controlar e gerenciar um sistema híbrido de geração de energia.

\section{REFERÊNCIAS}

BAGUL, A. D., Sizing of a stand-alone hybrid wind-photovoltaic system using a three-event probability density approximation. Solar Energy, v4, n.56, p.323-35, 1996.

BELLARMine, T. G., Wind energy for the 1990s and beyond. Energy Conversion and Management., v.12, n.37, p.1741-52, 1996.

MCNEILL, D., FREIBERGER, P, 1994, Fuzzy Logic. 1 ed. New York, EUA, Touchstone and Colophon.

MAHMOUD, M., Experience results and techno-economic feasibility of using photovoltaic generators instead of diesel motors for water pumping from rural desert wells in Jordan. IEE Proceedings, v.137, n.6, p.391-4, 1990.

SHAW, I. S., SIMÕES, M. G., 2001, Controle e Modelagem Fuzzy. 2 ed. São Paulo.

\section{SILVA, C.D. "Avaliação do Potencial das Energias Solar e Eólica para Acionamento de Pequenos}

Sistemas de Bombeamento na Fazenda Lageado". 2000. 98f. Dissertação (Mestrado em Agronomia Energia na Agricultura), Faculdade de Ciências Agronômicas, Universidade Estadual Paulista, Botucatu, 2000. 
SIQUEIRA, Jair Antonio Cruz. Desempenho de um sistema híbrido eólico-fotovoltaico de pequeno porte para energização rural. 2005. 176 f. Tese (Doutorado em Agronomia - Energia na Agricultura), Faculdade de Ciências Agronômicas, Universidade Estadual Paulista, Botucatu, 2005.

SOUZA, O. T. L. Desenvolvimento de um modelo fuzzy para determinação do calor latente com aplicação em sistemas de irrigação. 2004. 113 f. Tese (Doutorado em Agronomia - Energia na Agricultura), Faculdade de Ciências Agronômicas, Universidade Estadual Paulista, Botucatu, 2004. 